SLAC-PUB-8727

BABAR-PROC-00/37

December, 2000

\title{
First Physics Results at BABAR
}

\author{
Francesca Di Lodovico \\ University of Edinburgh \\ Dept. of Physics \\ James Clerk Maxwell Bldg., King's Bldgs \\ Mayfield Road \\ Edinburgh EH9 3JZ, Scotland, UK \\ (for the BABAR Collaboration)
}

The BABAR detector, which operates at the SLAC PEP-II asymmetric $e^{+} e^{-}$collider at energies near the $\Upsilon(4 S)$ resonance, started operation on the $26^{\text {th }}$ of May 1999 . We present the first study of $\sin 2 \beta$, with samples of $B^{0} \rightarrow J / \psi K_{S}^{0}$ and $B^{0} \rightarrow \psi(2 S) K_{S}^{0}$ decays, using $9.0 \mathrm{fb}^{-1}$ of data recorded between January and July 2000 at the $\Upsilon(4 S)$ resonance and $0.8 \mathrm{fb}^{-1}$ recorded $40 \mathrm{MeV}$ below the $\Upsilon(4 S)$ resonance. A preliminary result of $\sin 2 \beta=$ $0.12 \pm 0.37$ (stat) \pm 0.09 (syst) was obtained. Details of the analysis are given. Moreover, we present measurements of charged and neutral $B$ meson lifetimes and $B^{0} \bar{B}^{0}$ oscillation frequency.

Contributed to the Proceedings of the $5^{\text {th }}$ Heavy Quarks at Fixed Target Conference, 10/09/2000 - 10/12/2000, Rio de Janeiro, Brazil

Stanford Linear Accelerator Center, Stanford University, Stanford, CA 94309

Work supported in part by Department of Energy contract DE-AC03-76SF00515. 


\section{Introduction}

The primary goal of the BABAR experiment at PEP-II is to overconstrain the Unitarity Triangle. The sides of this triangle can be measured through non- $C P$ violating physics, such as $V_{u b}, V_{c b}, V_{t d}$ measurements [1], while its angles are accessible through $C P$ violating processes [1].

\section{PEP-II}

The PEP-II $B$ Factory [2] is an $e^{+} e^{-}$colliding beam storage ring complex on the SLAC site designed to produce a luminosity of at least $3 \times 10^{33} \mathrm{~cm}^{-2} \mathrm{~s}^{-1}$ at a center-of-mass energy of $10.58 \mathrm{GeV}$, the mass of the $\Upsilon(4 S)$ resonance. In the 2000 run, the achieved average luminosity was $2.5 \times 10^{33} \mathrm{~cm}^{-2} \mathrm{~s}^{-1}$, with a daily average integrated luminosity of $135 \mathrm{pb}^{-1}$. The total collected luminosity was about $22 \mathrm{fb}^{-1}$. The machine is asymmetric with a High Energy Ring (HER) for the $9.0 \mathrm{GeV}$ electron beam and a Low Energy Ring (LER) for the $3.1 \mathrm{GeV}$ positron beam. This corresponds to $\beta \gamma=0.56$ and makes it possible to measure time dependent $C P$ violating asymmetries. It corresponds to an average separation of $\beta \gamma \mathrm{c} \tau=250 \mu \mathrm{m}$ between the two B mesons vertices.

\section{$3 \quad B A B A R$}

\subsection{Detector description [2]}

The volume within the BABAR superconducting solenoid, which produces a $1.5 \mathrm{~T}$ axial magnetic field, consists of: a five layer silicon strip vertex detector (SVT), a central drift chamber (DCH), a quartz-bar Cherenkov radiation detector (DIRC) and a CsI crystal electromagnetic calorimeter (EMC). Two layers of cylindrical resistive plate counters (RPCs) are located between the barrel calorimeter and the magnet cryostat. All the detectors located inside the magnet have full acceptance in azimuth. The integrated flux return (IFR) outside the cryostat is composed of 18 layers of steel, which successively increase in thickness away from the interaction point, and are instrumented with 19 layers of planar RPCs in the barrel and 18 in the endcaps.

\subsection{Event reconstruction [2]}

Charged particles are detected and their momentum is measured by a combination of the DCH and SVT. The charged particle momentum resolution is approximately given by $\left(\delta p_{T} / p_{T}\right)^{2}=\left(0.0015 p_{T}\right)^{2}+(0.005)^{2}$, where $p_{T}$ is in $\mathrm{GeV} / c$. The SVT, with a typical resolution of $10 \mu \mathrm{m}$ per hit, provides excellent vertex resolution both in the transverse plane and in $z$. The vertex resolution in $z$ is typically $50 \mu \mathrm{m}$ for a fully reconstructed $B$ meson and of order $100 \mu \mathrm{m}$ for the distance among the two $B$ mesons when only one is fully reconstructed. Leptons and hadrons are identified using a combination of measurements from all the BABAR components, including the energy loss $\mathrm{d} E / \mathrm{d} x$ in the helium-based gas of the DCH (40 samples maximum) and in the silicon of the SVT (5 samples maximum). Electrons and photons are identified in the barrel and the forward regions by the EMC, and muons are 
identified in the IFR. In the barrel region the DIRC provides excellent kaon identification over the full momentum range above $250 \mathrm{MeV} / \mathrm{c}$.

\section{$4 \sin 2 \beta$ measurement}

In $e^{+} e^{-}$storage rings operating at the $\Upsilon(4 S)$ resonance a $B^{0} \bar{B}^{0}$ pair produced in a $\Upsilon(4 S)$ decay evolves in a coherent $P$-wave until one of the $B$ mesons decays. If one of the $B$ mesons $\left(B_{\text {tag }}\right)$ can be ascertained to decay to a state of known flavor at a certain time $t_{\text {tag }}$, the other $B\left(B_{C P}\right)$ is at that time known to be of the opposite flavor. For the measurement of $\sin 2 \beta, B_{C P}$ is fully reconstructed in a $C P$ eigenstate $\left(J / \psi K_{S}^{0}\right.$ or $\left.\psi(2 S) K_{S}^{0}\right)$. By measuring the proper time interval $\Delta t=t_{C P}-t_{t a g}$ from the $B_{t a g}$ decay time to the decay of the $B_{C P}$ $\left(t_{C P}\right)$, it is possible to determine the time evolution of the initially pure $B^{0}$ or $\bar{B}^{0}$ state:

$$
f_{ \pm}\left(\Delta t ; \Gamma, \Delta m_{d}, \mathcal{D} \sin 2 \beta\right)=\frac{1}{4} \Gamma \mathrm{e}^{-\Gamma|\Delta t|}\left[1 \pm \mathcal{D} \sin 2 \beta \times \sin \Delta m_{d} \Delta t\right]
$$

where the + or - sign indicates whether the $B_{\text {tag }}$ is tagged as a $B^{0}$ or a $\bar{B}^{0}$, respectively. The dilution factor $\mathcal{D}$ is given by $\mathcal{D}=1-2 w$, where $w$ is the mistag fraction, i.e., the probability that the flavor of the tagging $B$ is identified incorrectly. A direct $C P$ violation term proportional to $\cos \Delta m_{d} \Delta t$ could arise from the interference between two decay mechanisms with different weak phases. In the Standard Model, we consider that the dominant diagrams for the decay modes have no relative weak phase, so no such term is expected.

To account for the finite resolution of the detector, the time-dependent distributions $f_{ \pm}$ for $B^{0}$ and $\bar{B}^{0}$ tagged events (Eq. I) must be convoluted with a time resolution function $\mathcal{R}(\Delta t ; \hat{a})$ :

$$
\mathcal{F}_{ \pm}\left(\Delta t ; \Gamma, \Delta m_{d}, \mathcal{D} \sin 2 \beta, \hat{a}\right)=f_{ \pm}\left(\Delta t ; \Gamma, \Delta m_{d}, \mathcal{D} \sin 2 \beta\right) \otimes \mathcal{R}(\Delta t ; \hat{a}),
$$

where $\hat{a}$ represents the set of parameters that describe the resolution function.

It is possible to construct a $C P$-violating observable

$$
\mathcal{A}_{C P}(\Delta t)=\frac{\mathcal{F}_{+}(\Delta t)-\mathcal{F}_{-}(\Delta t)}{\mathcal{F}_{+}(\Delta t)+\mathcal{F}_{-}(\Delta t)},
$$

which is approximately proportional to $\sin 2 \beta$ :

$$
\mathcal{A}_{C P}(\Delta t) \sim \mathcal{D} \sin 2 \beta \times \sin \Delta m_{d} \Delta t
$$

Since no time-integrated $C P$ asymmetry effect is expected, an analysis of the time-dependent asymmetry is necessary. At an asymmetric-energy $B$ Factory, the proper decay-time difference $\Delta t$ is, to an excellent approximation, proportional to the distance $\Delta z$ between the two $B^{0}$-decay vertices along the axis of the boost, $\Delta t \approx \Delta z / \mathrm{c}\langle\beta \gamma\rangle$.

Since the amplitude of the time-dependent $C P$-violating asymmetry in Eq. 1 involves the product of $\mathcal{D}$ and $\sin 2 \beta$, one needs to determine the dilution factors $\mathcal{D}_{i}$ (or equivalently the mistag fractions $w_{i}$ ) in order to extract the value of $\sin 2 \beta$. The mistag fractions are determined from the data by studying the time-dependent rate of $B^{0} \bar{B}^{0}$ oscillations. 

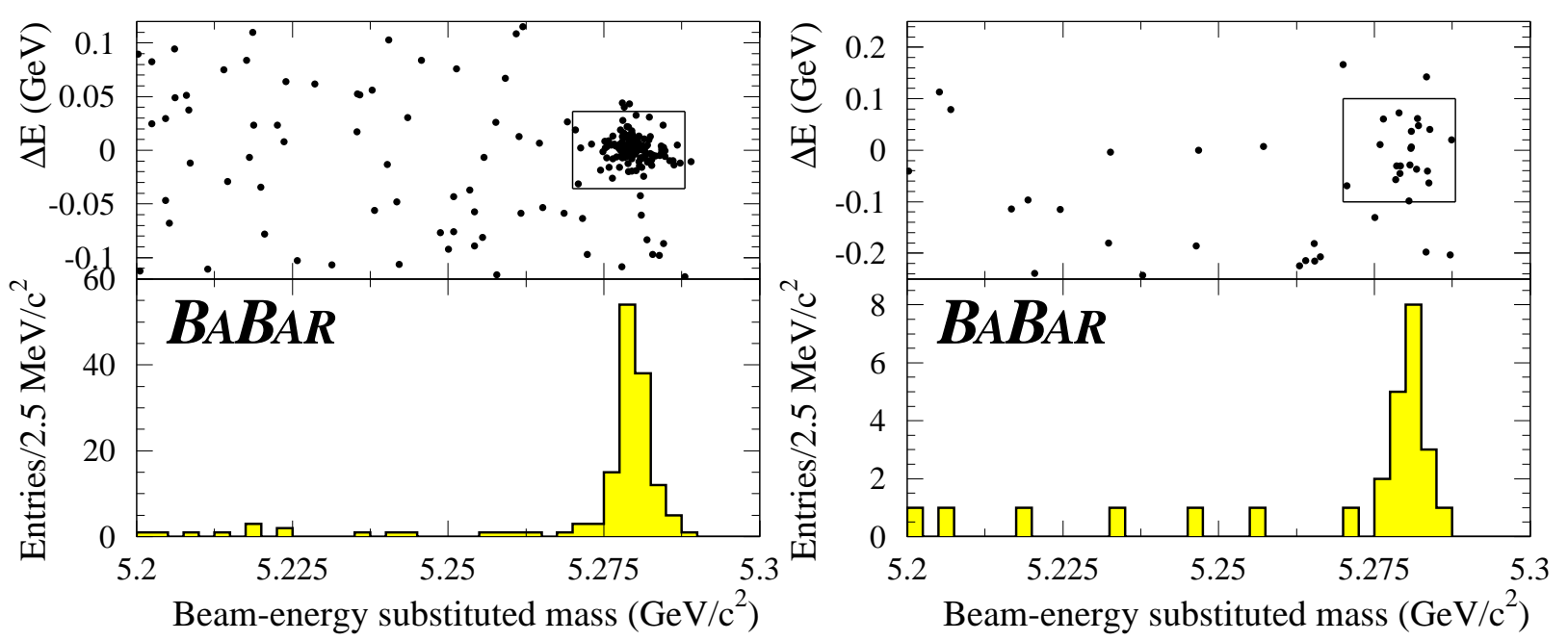

Figure 1: $J / \psi K_{S}^{0}\left(K_{S}^{0} \rightarrow \pi^{+} \pi^{-}\right)$signal (left). $J / \psi K_{S}^{0}\left(K_{S}^{0} \rightarrow \pi^{0} \pi^{0}\right)$ signal (right).

The value of the single free parameter $\sin 2 \beta$ is extracted from the tagged $B_{C P}$ sample by maximizing the likelihood function

$\ln \mathcal{L}_{C P}=\sum_{i}\left[\sum_{B^{0} \operatorname{tag}} \ln \mathcal{F}_{+}\left(\Delta t ; \Gamma, \Delta m_{d}, \hat{a}, \mathcal{D}_{i} \sin 2 \beta\right)+\sum_{\bar{B}^{0} \operatorname{tag}} \ln \mathcal{F}_{-}\left(\Delta t ; \Gamma, \Delta m_{d}, \hat{a}, \mathcal{D}_{i} \sin 2 \beta\right)\right]$

where the outer summation is over tagging categories $i$.

\subsection{Analysis}

For this analysis we use a sample of $9.8 \mathrm{fb}^{-1}$ of data recorded between January and the beginning of July 2000, of which $0.8 \mathrm{fb}^{-1}$ was recorded $40 \mathrm{MeV}$ below the $\Upsilon(4 S)$ resonance (off-resonance data).

The measurement of the $C P$-violating asymmetry has five main components :

- Selection of the signal $B^{0} / \bar{B}^{0} \rightarrow J / \psi K_{S}^{0}$ and $B^{0} / \bar{B}^{0} \rightarrow \psi(2 S) K_{S}^{0}$ events, as described in detail in [3].

Distributions of $\Delta E$, the difference between the reconstructed and expected $B$ meson energy measured in the center-of-mass frame, and $m_{\mathrm{ES}}$, the beam-energy substitued mass, are shown in Fig. 1 for the $J / \psi K_{S}^{0}\left(K_{S}^{0} \rightarrow \pi^{+} \pi^{-}\right)$and $J / \psi K_{S}^{0}\left(K_{S}^{0} \rightarrow \pi^{0} \pi^{0}\right)$ samples. Signal event yields and purities, determined from a fit to the $m_{\mathrm{ES}}$ distributions after selection on $\Delta E$, are summarized in Table $\mathbb{1}$.

- Measurement of the distance $\Delta z$ between the two $B^{0}$ decay vertices along the $\Upsilon(4 S)$ boost axis, as described in detail in [4] and [5]. 
Table 1: Event yields for the different samples used in this analysis, from the fit to $m_{\mathrm{ES}}$ distributions after selection on $\Delta E$. The purity is quoted for $m_{\mathrm{ES}}>5.270 \mathrm{MeV} / c^{2}$.

\begin{tabular}{|l|c|c|}
\hline Final state & Yield & Purity (\%) \\
\hline \hline$J / \psi K_{S}^{0}\left(K_{S}^{0} \rightarrow \pi^{+} \pi^{-}\right)$ & $124 \pm 12$ & 96 \\
$J / \psi K_{S}^{0}\left(K_{S}^{0} \rightarrow \pi^{0} \pi^{0}\right)$ & $18 \pm 4$ & 91 \\
$\psi(2 S) K_{S}^{0}$ & $27 \pm 6$ & 93 \\
\hline
\end{tabular}

From the measurement of $\Delta z, \Delta t$ can be computed.

The time resolution function is described accurately by the sum of two Gaussian distributions, which has five independent parameters:

$$
\mathcal{R}(\Delta t ; \hat{a})=\sum_{i=1}^{2} \frac{f_{i}}{\sigma_{i} \sqrt{2 \pi}} \exp \left(-\left(\Delta t-\delta_{i}\right)^{2} / 2 \sigma_{i}{ }^{2}\right) .
$$

A fit to the time resolution function in Monte Carlo simulated events indicates that most of the events $\left(f_{1}=1-f_{2}=70 \%\right)$ are in the core Gaussian, which has a width $\sigma_{1} \approx 0.6 \mathrm{ps}$. The wide Gaussian has a width $\sigma_{2} \approx 1.8 \mathrm{ps}$. Tracks from forward-going charm decays included in the reconstruction of the $B_{t a g}$ vertex introduce a small bias, $\delta_{1} \approx-0.2 \mathrm{ps}$, for the core Gaussian.

- Determination of the flavor of the $B_{\text {tag }}$, as described in detail in [4].

Each event with a $C P$ candidate is assigned a $B^{0}$ or $\bar{B}^{0}$ tag if the rest of the event (i.e., with the daughter tracks of the $B_{C P}$ removed) satisfies the criteria from one of several tagging categories. In other words, a $B^{0}$ tag indicates that the $B_{C P}$ candidate was in a $\bar{B}^{0}$ state at $\Delta t=0$; a $\bar{B}^{0}$ tag indicates that the $B_{C P}$ candidate was in a $B^{0}$ state.

Three tagging categories rely on the presence of a fast lepton (Lepton categories) and/or one or more charged kaons in the event (Kaon category). Two categories, called neural network categories (NT1 and NT2), are based upon the output value of a neural network algorithm applied to events that have not already been assigned to lepton or kaon tagging categories.

- Measurement of the dilution factors $\mathcal{D}_{i}$ from the data for the different tagging categories, as described in detail in [4].

The figure of merit for each tagging category is the effective tagging efficiency $Q_{i}=$ $\varepsilon_{i}\left(1-2 w_{i}\right)^{2}$, where $\varepsilon_{i}$ is the fraction of events assigned to category $i$ and $w_{i}$ is the mistag fraction.

The mistag fractions are measured directly in events in which one $B^{0}$ candidate, called the $B_{r e c}$, is fully reconstructed in a flavor eigenstate mode. The flavor-tagging algorithms are applied to the rest of the event, which constitutes the potential $B_{\text {tag }}$, assuming the $B_{r e c}$ is properly tagged.

The mistag fractions and the tagging efficiencies obtained by combining the results from maximum likelihood fits to the time distributions in the $B^{0}$ hadronic and semileptonic samples are summarized in Table 2 . 
Table 2: Mistag fractions measured from a maximum-likelihood fit to the time distribution for the fully-reconstructed $B^{0}$ sample. The Electron and Muon categories are grouped into one Lepton category. The uncertainties on $\varepsilon$ and $Q$ are statistical only.

\begin{tabular}{|l|c|c|c|}
\hline Tagging Category & $\varepsilon(\%)$ & $w(\%)$ & $Q(\%)$ \\
\hline \hline Lepton & $11.2 \pm 0.5$ & $9.6 \pm 1.7 \pm 1.3$ & $7.3 \pm 0.7$ \\
Kaon & $36.7 \pm 0.9$ & $19.7 \pm 1.3 \pm 1.1$ & $13.5 \pm 1.2$ \\
NT1 & $11.7 \pm 0.5$ & $16.7 \pm 2.2 \pm 2.0$ & $5.2 \pm 0.7$ \\
NT2 & $16.6 \pm 0.6$ & $33.1 \pm 2.1 \pm 2.1$ & $1.9 \pm 0.5$ \\
\hline \hline all & $76.7 \pm 0.5$ & & $27.9 \pm 1.6$ \\
\hline
\end{tabular}

Table 3: Summary of systematic uncertainties. The different contributions to the systematic error are added in quadrature.

\begin{tabular}{|l|c|}
\hline Source of uncertainty & Uncertainty on $\sin 2 \beta$ \\
\hline \hline uncertainty on $\tau_{B}^{0}$ & 0.002 \\
uncertainty on $\Delta m_{d}$ & 0.015 \\
uncertainty on $\Delta z$ resolution for $C P$ sample & 0.019 \\
uncertainty on time-resolution bias for $C P$ sample & 0.047 \\
uncertainty on measurement of mistag fractions & 0.053 \\
different mistag fractions for $C P$ and non- $C P$ samples & 0.050 \\
different mistag fractions for $B^{0}$ and $\bar{B}^{0}$ & 0.005 \\
background in $C P$ sample & 0.015 \\
\hline \hline total systematic error & $\mathbf{0 . 0 9 1}$ \\
\hline
\end{tabular}

- Extraction of the amplitude of the $C P$ asymmetry and the value of $\sin 2 \beta$ with an unbinned maximum likelihood fit.

The maximum-likelihood fit for $\sin 2 \beta$, using the full tagged sample of $B^{0} / \bar{B}^{0} \rightarrow J / \psi K_{S}^{0}$ and $B^{0} / \bar{B}^{0} \rightarrow \psi(2 S) K_{S}^{0}$ events, gives:

$$
\sin 2 \beta=0.12 \pm 0.37 \text { (stat) } \pm 0.09 \text { (syst) }
$$

For this result, the $B^{0}$ lifetime and $\Delta m_{d}$ are fixed to the current best values [6]. The log likelihood is shown as a function of $\sin 2 \beta$ and the $\Delta t$ distributions for $B^{0}$ and $\bar{B}^{0}$ tags are shown in Fig. 2. The contributions to the systematic uncertainty are summarized in Table 3. They are added in quadrature to obtain the total systematic error.

Improvements in the $\sin 2 \beta$ measurement are expected in the near future with the accumulation and analysis of more data and further systematic studies. 

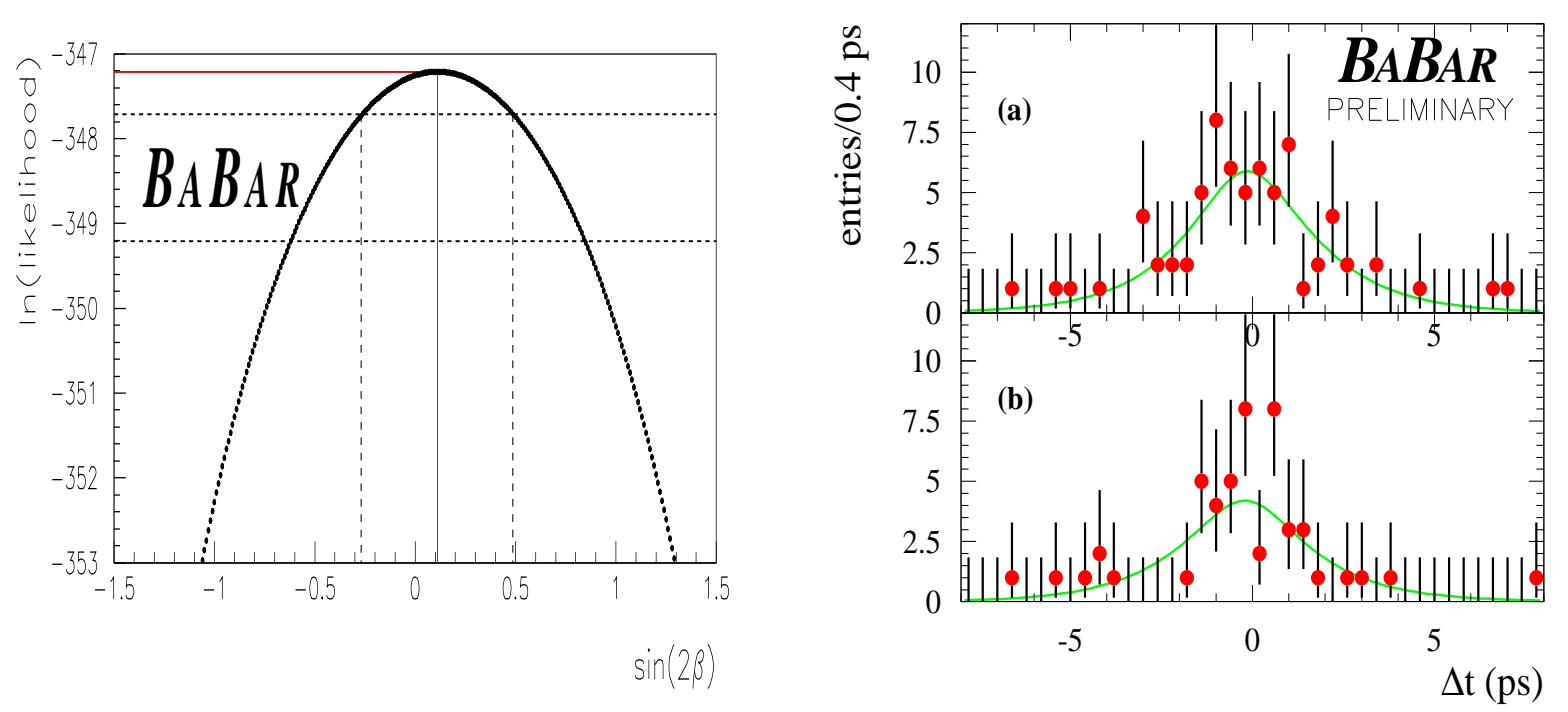

Figure 2: Variation of the log likelihood as a function of $\sin 2 \beta$ (left). The two horizont al dashed lines indicate changes in the log likelihood corresponding to one and two statistical standard deviations. Distribution of $\Delta t$ for (a) the $B^{0}$ tagged events and (b) the $\bar{B}^{0}$ tagged events in the $C P$ sample (right).

\section{Measurements of charged and neutral $B$ meson lifetimes and $B^{0} \bar{B}^{0}$ oscillations}

These measurements can be used to test theoretical models of heavy-quark decays and to constrain the Unitarity Triangle (via the sensitivity to the value of the CKM matrix element $\left.V_{t d}\right)$.

One $B\left(B_{r e c}\right)$ is fully reconstructed in an all-hadronic $\left(B^{0} \rightarrow D^{(*)-} \pi^{+}, D^{(*)-} \rho^{+}, D^{(*)-} a_{1}^{+}\right.$, $J / \psi K^{* 0}$ and $\left.B^{+} \rightarrow \bar{D}^{(*) 0} \pi^{+}, J / \psi K^{+}, \psi(2 S) K^{+}\right)$or semileptonic decay mode $\left(B^{0} \rightarrow D^{*-} \ell^{+} \nu\right)$ 凹. A total of about $2600 B^{0}$ and a similar number of charged $B$ are reconstructed in the hadronic modes, with a mean purity of $\sim 90 \%$. The background is mainly combinatorial. About 7500 $B^{0}$ are reconstructed in the semileptonic modes, with a mean purity of $\sim 84 \%$. Backgrounds to the semi-leptonic mode are due to combinatorics, wrong leptons, $c \bar{c}$ events, and charged $B$ decays from $B^{+} \rightarrow D^{* * 0} l^{+} \nu$.

The separation between the two $B$ vertices $\left(z_{\text {rec }}\right.$ and $z_{T A G}$ for the reconstructed and tagged vertex, respectively) along the boost direction, $\Delta z=z_{\text {rec }}-z_{\text {tag }}$, is measured and used to estimate the decay time difference, $\Delta t=\Delta z / \beta_{z} c$. The $\Delta t$ resolution is dominated by the precision on the $B_{\text {tag }}$ vertex, and has little dependence on the decay mode of the $B_{\text {rec }}$.

\footnotetext{
${ }^{1}$ Throughout this paper, conjugate modes are implied.
} 

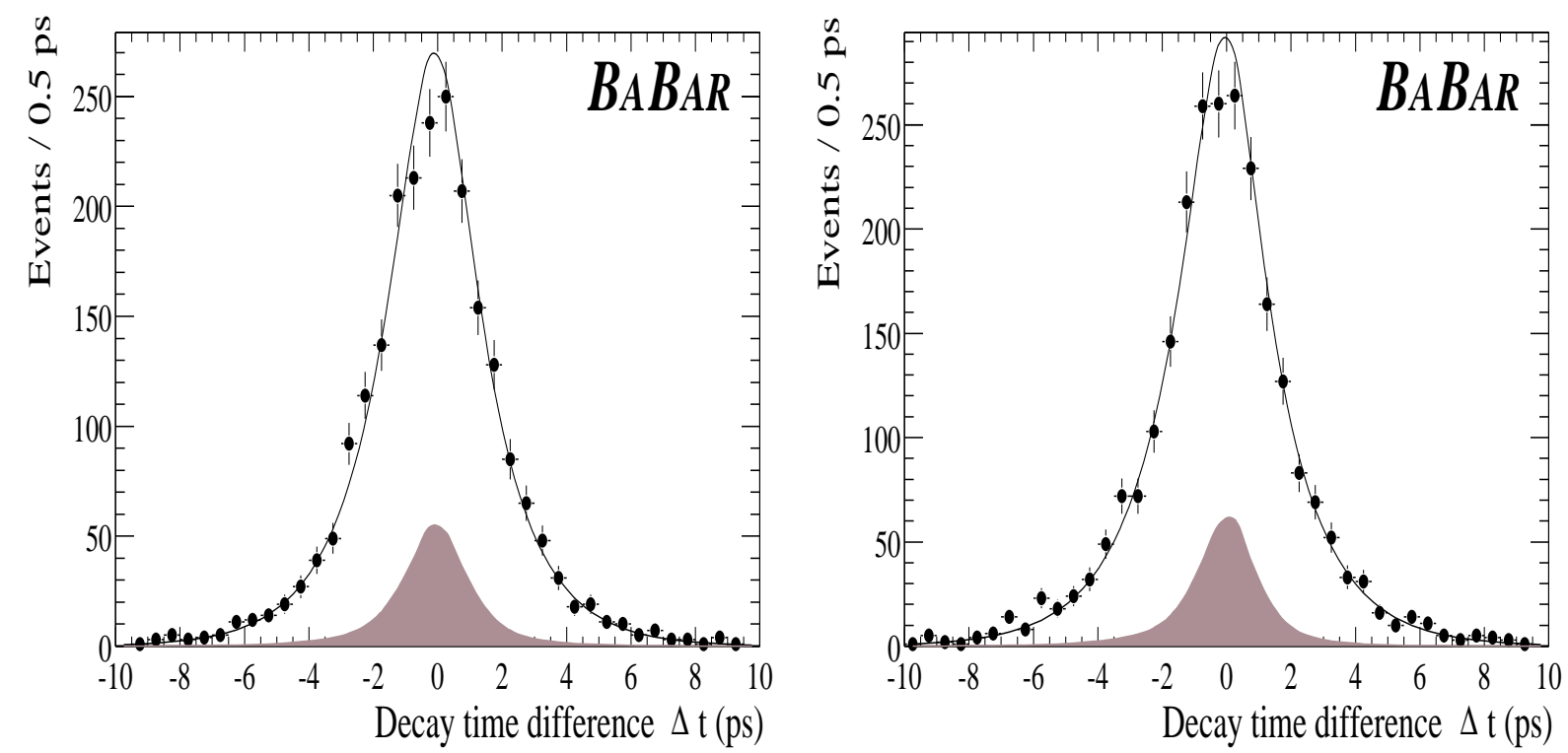

Figure 3: $\Delta t$ distributions for $B^{0} / \bar{B}^{0}$ (left) and $B^{+} / B^{-}$(right) candidates in the signal region $\left(m_{\mathrm{ES}}>5.27 \mathrm{GeV} / c^{2}\right)$. The result of the lifetime fit is superimposed. The background is shown by the hatched area.

\subsection{Lifetime Measurements}

The $B^{0}$ and $B^{+}$lifetimes are extracted from a simultaneous unbinned maximum likelihood fit to the $\Delta t$ distributions of the signal candidates, assuming a common resolution function. Only hadronic modes have been used. An empirical description of the $\Delta t$ background shape is assumed, using $m_{\mathrm{ES}}$ sidebands with independent parameters for neutral and charged mesons. Fig. 3 shows the $\Delta t$ distributions with the fit result superimposed.

\subsection{Time-dependent $B^{0} \bar{B}^{0}$ mixing}

A time-dependent $B^{0} \bar{B}^{0}$ mixing measurement requires the determination of the flavor of both $B$ mesons. Considering the $B^{0} \bar{B}^{0}$ system as a whole, one can classify the tagged events as mixed or unmixed depending on whether the $B_{t a g}$ is tagged with the same flavor as the $B_{\text {rec }}$ or with the opposite flavor.

From the time-dependent rate of mixed $\left(N_{\text {mix }}\right)$ and unmixed $\left(N_{\text {unmix }}\right)$ events, the mixing asymmetry $a(\Delta t)=\left(N_{\text {unmix }}-N_{\text {mix }}\right) /\left(N_{\text {unmix }}+N_{\text {mix }}\right)$ is calculated as a function of $\Delta t$ and fit to the expected cosine distribution. A simultaneous unbinned likelihood fit to the mixing asymmetry frequency and its amplitude allows the determination of both $\Delta m_{d}$ and the mistag rates, $w_{i}$, for the different tagging categories. The fit is performed simultaneously in each tagging category, assuming a common resolution function. Fig. 1 shows the $a(\Delta t)$ distributions with the fit result superimposed. 

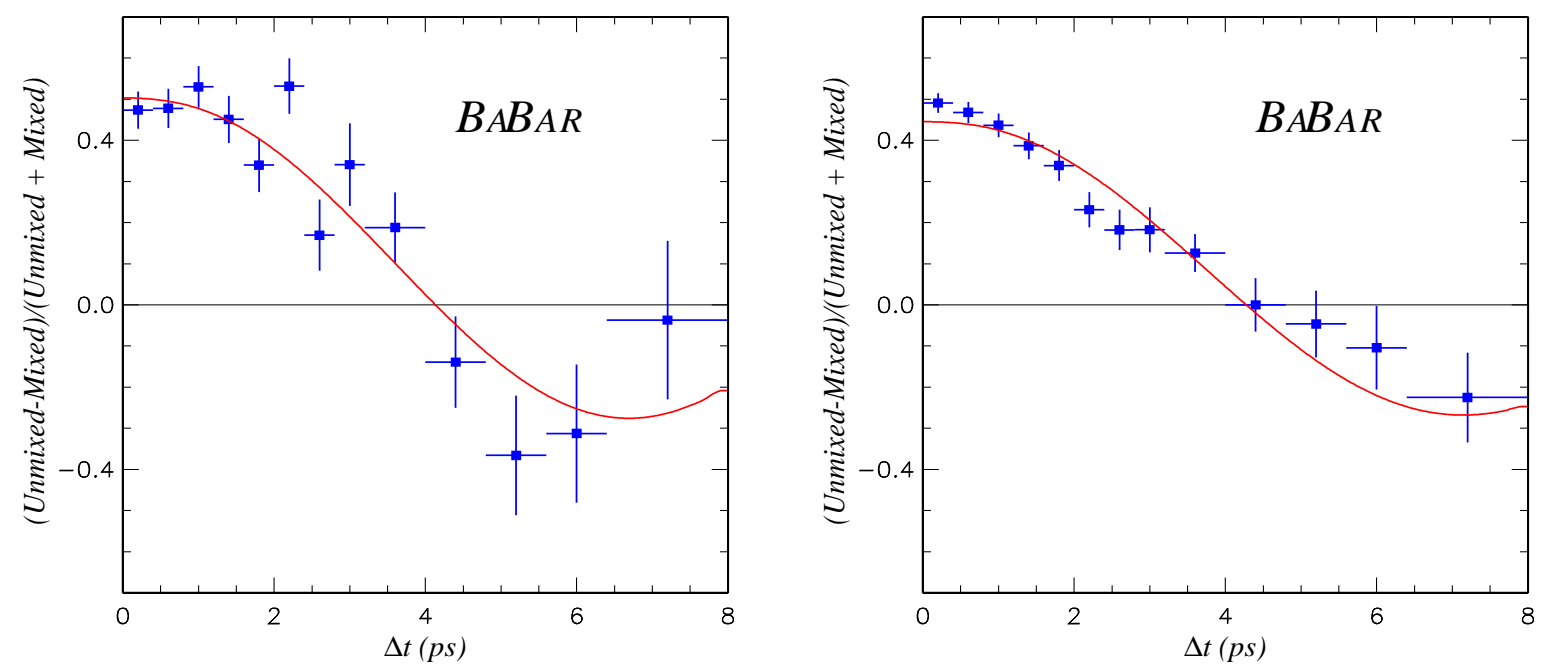

Figure 4: Time-dependent asymmetry $a(\Delta t)$ between unmixed and mixed events for (left) hadronic $B$ candidates with $m_{\mathrm{ES}}>5.27 \mathrm{GeV} / c^{2}$ and (right) for $B \rightarrow D^{*} \ell \nu$ candidates.

\section{$5.3 \quad$ Results}

The preliminary results for the $B$ meson lifetimes are $\tau_{B^{0}}=1.506 \pm 0.052$ (stat) \pm 0.029 (syst) ps and $\tau_{B^{+}}=1.602 \pm 0.049$ (stat) \pm 0.035 (syst) ps and for their ratio is $\tau_{B^{+}} / \tau_{B^{0}}=1.065 \pm$ 0.044 (stat) \pm 0.021 (syst).

From the hadronic $B^{0}$ sample we measure the $B^{0} \bar{B}^{0}$ oscillation frequency: $\Delta m_{d}=0.516 \pm$ 0.031 (stat) \pm 0.018 (syst) $\hbar \mathrm{ps}^{-1}$ and from the $D^{*-} \ell^{+} \nu$ sample the result is $\Delta m_{d}=0.508 \pm$ 0.020 (stat) \pm 0.022 (syst) $\hbar \mathrm{ps}^{-1}$ Combining the two $\Delta m_{d}$ results, we obtain the preliminary result: $\Delta m_{d}=0.512 \pm 0.017$ (stat) \pm 0.022 (syst) $\hbar \mathrm{ps}^{-1}$. The mistag rates and $\Delta t$ resolution function extracted from these fits to the data are used in the BABAR CP violation asymmetry analysis [7]. The above results are consistent with previous measurements [6] and are of similar precision. The mixing result is compatible with a BABAR measurement using dileptons [8].

\section{Conclusions}

We have presented $B A B A R$ 's first measurement of the $C P$-violating asymmetry parameter $\sin 2 \beta$ in the $B$ meson system:

$$
\sin 2 \beta=0.12 \pm 0.37 \text { (stat) } \pm 0.09 \text { (syst) }
$$

Our measurement is consistent with the world average $\sin 2 \beta=0.9 \pm 0.4$ [6], and is currently limited by the size of the $C P$ sample.

We have presented also the time-dependent mixing and lifetime measurements, performed for the first time at the $\Upsilon(4 S)$. 
Other important competitive measurements have been achieved by BABAR.

All measurement are foreseen to be improved in the near future, with the accumulation and study of more data and further systematic studies.

\section{References}

[1] P. H. Harrison and H. R. Quinn, eds., "The BABAR physics book",

[2] BABAR Collaboration, B. Aubert et al., "The first year of the BABAR experiment at PEP-II", hep-ex/0008059.

[3] BABAR Collaboration, B. Aubert et al., "Exclusive $B$ decays to charmonium final states", hep-ex/0008050.

[4] BABAR Collaboration, B. Aubert et al., "A measurement of the $B^{0} \bar{B}^{0}$ oscillation frequency and determination of flavor-tagging efficiency using semileptonic and hadronic $B$ decays", hep-ex/0008052.

[5] BABAR Collaboration, B. Aubert et al., "A measurement of the charged and neutral $B$ meson lifetimes using fully reconstructed decays", hep-ex/0008060.

[6] Particle Data Group, D. E. Groom et al., Eur. Phys. Jour. C 15, 1 (2000).

[7] BABAR Collaboration, B. Aubert et al. "A study of time-dependent $C P$-asymmetries in $B^{0} \rightarrow J / \psi K_{S}^{0}$ and $B^{0} \rightarrow \psi(2 S) K_{S}^{0}$ decays", hep-ex/0008048.

[8] BABAR Collaboration, B. Aubert et al., "Measurement of the time dependence of $B^{0} \bar{B}^{0}$ oscillations using inclusive dilepton events", hep-ex/0008054. 